\title{
Contrôle de croissance des ovins allaitants en Tunisie. II. Perte de précision sur le calcul des performances à âge type selon un protocole simplifié alternatif au protocole officiel
}

\author{
M. Ben Hamouda ${ }^{*}$ M. Rekik ${ }^{2}$
}

\begin{abstract}
Mots-clés
Ovin - Poids à la naissance - Contrôle de croissance - Méthode alternative Tunisie.
\end{abstract}

\begin{abstract}
Résumé
Les performances à âge type P10 (poids à 10 jours), P30, P70, GMQ10/30 (gains moyens quotidiens entre 10 et 30 jours) et GMQ30/70 de 9642 agneaux de race Barbarine ont été calculées selon le protocole conventionnel "F2 », ou un protocole simplifié, basé sur l'ajustement de la fonction von Bertalanffy à deux pesées à 42 jours d'intervalle, la première ayant lieu entre 2 et 60 jours après la naissance de l'agneau, et un poids à la naissance réel (PNR : contrôlé à la naissance), fixé, le même pour tous les agneaux $(P N F=3,4 \mathrm{~kg}$ ) ou estimé en fonction de la première pesée $(\mathrm{P})$ et l'intervalle entre la naissance et la première pesée $(\mathrm{D})[\mathrm{PNE}=2,71009+0,208313$ $\left.P-0,00578 P(60-D)+0,00016 P(60-D)^{2}\right]$. La perte de précision moyenne associée au calcul des poids à âge type a été élevée pour P10 (40 à 45 p. 100), modérée pour P30 (6 à 7 p. 100) et faible pour P70 (2 à 3 p. 100). La perte de précision associée au GMQ10/30 a été inférieure à celle déjà admise par le protocole conventionnel pour un intervalle entre la naissance et le premier contrôle compris entre 15 et 45 jours (15 p. 100 au maximum), et du même ordre entre 7 et 15 jours d'intervalle (20 p. 100 au maximum). Ces résultats ont permis de proposer la simplification du protocole F2 à deux ou trois pesées et un poids à la naissance. La première pesée devrait être retardée jusqu'à 46 jours après la naissance alors que l'intervalle entre contrôles successifs peut varier entre 30 et 40 jours, en fonction de la perte de précision pouvant être tolérée sur l'estimation des performances à âge type.
\end{abstract}

\section{INTRODUCTION}

Dans un schéma d'amélioration génétique, l'opération la plus importante est le contrôle des performances qui doit être «simple, fiable et peu coûteux » (9). Avec l'évolution de la conjoncture économique liée à la mondialisation, l'éleveur doit chercher par tous les moyens à réduire les coûts afin de faire face à une concurrence de plus en plus rude, notamment dans la filière des petits

\footnotetext{
1. Institution de la recherche et de l'enseignement supérieur agricoles, 30 rue Alain Savary, 1002 Tunis, Tunisie.

Tél. : +21698385634; fax : +21671 796170

E-mail : m.b.hammouda@iresa.agrinet.tn

2. Ecole nationale de médecine vétérinaire, Sidi Thabet, Tunisie

* Auteur pour la correspondance
}

ruminants dont l'avenir est jugé incertain (7). Pour alléger les charges, de nombreux travaux de recherche ont envisagé de simplifier les protocoles de contrôle laitier $(1,6,8,11)$ et de croissance $(2,13)$ des ovins. En Tunisie, le contrôle de croissance des ovins allaitants nécessite au moins cinq à six visites d'un technicien à 21 jours d'intervalle, ce qui est très onéreux. En outre, le principe de la méthode conventionnelle ne semble pas le mieux approprié aux conditions d'élevage extensif des régions arides et semi-arides (4). Dans ce cadre et en continuité avec des travaux antérieurs (3, 4), cette étude a eu comme objectifs principaux (i) d'estimer les poids à âge type en ajustant une courbe de croissance curvilinéaire utilisant un nombre réduit de contrôles, (ii) de mesurer les conséquences de la simplification du nombre de contrôles sur la précision de l'estimation des poids à âge type, et (iii) de proposer de nouveaux protocoles de contrôle de croissance pour les ovins allaitants adaptés aux conditions difficiles. 


\section{MATERIEL ET METHODES}

\section{Données}

Les données ayant servi de support au présent travail sont celles décrites par Ben Hamouda (4). Il s'agit des 9642 agneaux de race Barbarine des deux troupeaux de la station expérimentale d'Ousseltia (Inrat, Tunisie) contrôlés pour la croissance entre 1969 et 1998, pesés à la naissance, puis six fois à intervalles de 21 jours.

\section{Méthodes}

Dans une première étude, Ben Hamouda (3) a constaté que l'utilisation d'un ajustement curvilinéaire des données ne peut être envisagée que si l'on y inclut la valeur du poids à la naissance (PN). Pour ce faire, on peut soit fixer un PN moyen pour tous les agneaux, soit utiliser un PN pour chaque catégorie établie selon la combinaison de sexe et de mode de naissance (3). En outre, ignorer un contrôle sur deux dans le protocole officiel actuel n'altèrerait pas la forme de la courbe de croissance et permettrait d'estimer les performances à âge type sans trop de perte de précision. Partant de ces remarques préliminaires, en se basant sur des ajustements selon la fonction de von Bertalanffy (4), les possibilités de simplification ci-après ont été étudiées

\section{Augmenter l'intervalle entre visites}

Le rythme des visites est actuellement d'une pesée tous les 21 jours. Il s'agissait d'ignorer un contrôle sur deux de manière à passer d'un intervalle moyen entre visites successives de 21 jours à un intervalle de 42 jours. Par ailleurs et afin de situer la première visite, par rapport à la date du début des agnelages dans un troupeau, trois scénarios sur les deux contrôles séquentiels retenus pour l'ajustement de la courbe ont été envisagés : (i) l'utilisation des premières et troisièmes pesées actuelles $\mathrm{P}_{1}$ et $\mathrm{P}_{3}$, soit une première visite effectuée à un intervalle naissance - premier contrôle variant entre 1 et 21 jours ; (ii) l'utilisation de $\mathrm{P}_{2}$ et $\mathrm{P}_{4}$, soit une première visite effectuée à un intervalle naissance - premier contrôle allant de 22 à 42 jours; et (iii) l'utilisation de $\mathrm{P}_{3}$ et $\mathrm{P}_{5}$, soit une première visite effectuée à un intervalle naissance - premier contrôle variant entre 43 et 63 jours, tronqué à 60 jours. Ces trois simulations ont ainsi permis d'étudier la simplification sur un intervalle entre la naissance et le premier contrôle allant de 1 à 60 jours. La répartition des effectifs relative à ce protocole est présentée dans le tableau I.

\section{Prise en compte du poids à la naissance}

L'utilisation nécessaire du poids à la naissance pour établir les courbes d'ajustement ne doit pas être en contradiction avec l'objectif de simplification du protocole de recueil d'information. A cette fin, les trois situations suivantes ont été simulées: (i) le poids à la naissance réel (PNR) pris comme situation de référence ; (ii) un poids à la naissance fixé, identique pour tous les agneaux $(\mathrm{PNF}=3,4)(4)$; et (iii) un poids à la naissance estimé, par régression sur la première pesée $(\mathrm{P})$ et l'intervalle naissance - premier contrôle $(\mathrm{D})$. La régression polynomiale quadratique ci-après a été utilisée pour estimer un poids à la naissance individuel (PNE) :

$\mathrm{PNE}=2,71009+0,208313 \mathrm{P}-0,00578 \mathrm{P}(60-\mathrm{D})+0,00016 \mathrm{P}$ $(60-\mathrm{D})^{2}$.

La perte de précision associée à l'estimation d'une performance, calculée par un protocole simplifié, est appréciée par le ratio 1- $\mathrm{R}^{2}$, $\mathrm{R}^{2}$ étant le coefficient de détermination du modèle de régression de cette performance par rapport à celle prise comme référence (4), et un coefficient de régression $« \mathrm{~b} »$ qui devrait avoisiner l'unité. A défaut de référence sur la perte de précision dans ce domaine, les taux déjà admis par la méthode conventionnelle (4) ont servi de témoin, notamment en ce qui concerne la valeur laitière ; sur l'intervalle de 1 à 62 jours, ce témoin est illustré par la figure 1.

Les analyses statistiques ont été effectuées par les procédures NLIN, REG et CORR du logiciel SAS 6.1 (12).

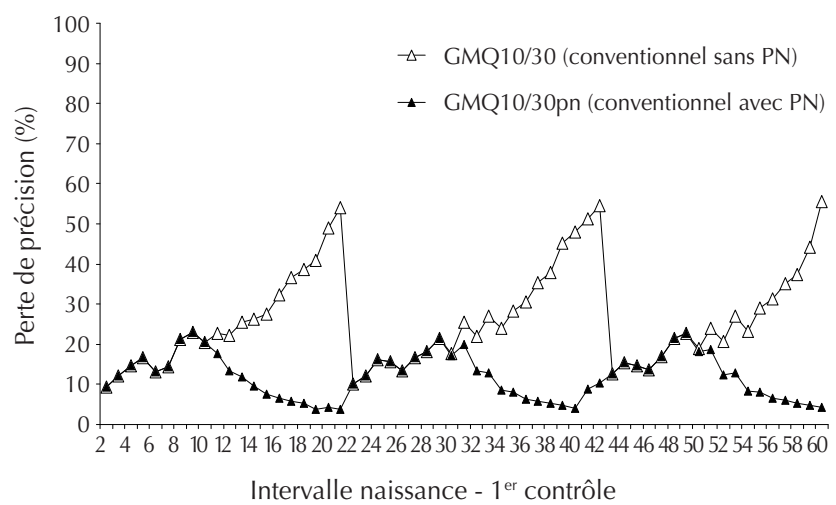

Figure 1 : perte de précision associée à l'estimation des GMQ10/30pn et GMQ10/30 par rapport au GMQ00/30 ( $P N$ : le premier contrôle est le poids à la naissance; GMQ10/30 : gains moyens quotidiens entre 10 et 30 jours).

\section{Tableau I}

Répartition des effectifs des agneaux contrôlés, pour la croissance, en fonction de l'intervalle naissance - $1^{\mathrm{er}}$ contrôle correspondant aux scénarios envisagés sur la première visite

\begin{tabular}{|c|c|c|c|c|c|}
\hline \multicolumn{2}{|c|}{ P1 - P3 } & \multicolumn{2}{|c|}{ P2 - P4 } & \multicolumn{2}{|c|}{ P3 - P5 } \\
\hline \multicolumn{2}{|c|}{$\begin{array}{l}\text { Naissance - Effectif } \\
1^{\text {er }} \text { contrôle }\end{array}$} & \multicolumn{2}{|c|}{$\begin{array}{l}\text { Naissance - Effectif } \\
1^{\text {er }} \text { contrôle }\end{array}$} & \multirow{2}{*}{$\begin{array}{c}\text { Naissance - } \\
\text { 1 }^{\text {er }} \text { contrôle } \\
43\end{array}$} & \multirow{2}{*}{$\begin{array}{c}\text { Effectif } \\
642\end{array}$} \\
\hline 1 & - & 22 & 566 & & \\
\hline 2 & 1240 & 23 & 659 & 44 & 603 \\
\hline 3 & 678 & 24 & 575 & 45 & 501 \\
\hline 4 & 558 & 25 & 505 & 46 & 490 \\
\hline 5 & 563 & 26 & 496 & 47 & 440 \\
\hline 6 & 504 & 27 & 454 & 48 & 361 \\
\hline 7 & 447 & 28 & 403 & 49 & 350 \\
\hline 8 & 380 & 29 & 364 & 50 & 392 \\
\hline 9 & 341 & 30 & 350 & 51 & 350 \\
\hline 10 & 351 & 31 & 374 & 52 & 409 \\
\hline 11 & 382 & 32 & 385 & 53 & 433 \\
\hline 12 & 387 & 33 & 433 & 54 & 488 \\
\hline 13 & 454 & 34 & 476 & 55 & 501 \\
\hline 14 & 476 & 35 & 516 & 56 & 571 \\
\hline 15 & 533 & 36 & 587 & 57 & 483 \\
\hline 16 & 545 & 37 & 487 & 58 & 487 \\
\hline 17 & 490 & 38 & 505 & 59 & 475 \\
\hline 18 & 499 & 39 & 437 & 60 & 226 \\
\hline 19 & 468 & 40 & 425 & & \\
\hline 20 & 202 & 41 & 371 & & \\
\hline 21 & 144 & 42 & 252 & & \\
\hline Total & 9642 & & 9620 & & 8202 \\
\hline
\end{tabular}

$P_{1}-P_{3}$ : intervalle naissance - $1^{\text {er }}$ contrôle entre 1 et 21 jours $\mathrm{P}_{2}-\mathrm{P}_{4}$ : intervalle naissance - $1^{\mathrm{er}}$ contrôle entre 22 et 42 jours $\mathrm{P}_{3}-\mathrm{P}_{5}$ : intervalle naissance - $1^{\mathrm{er}}$ contrôle entre 43 et 60 jours 


\section{RESULTATS}

L'application de la régression polynomiale quadratique aux données a permis d'obtenir un PNE de $3,22 \mathrm{~kg}$ avec un écart-type de $0,16 \mathrm{~kg}$; les valeurs réelles ont été respectivement de 3,39 et $0,69 \mathrm{~kg}$. Les taux globaux de perte de précision associés au calcul des performances à âge type par les différents protocoles simplifiés sont présentés dans le tableau II.

Dans un intervalle naissance - premier contrôle allant de 22 à 42 jours et quelle que soit l'hypothèse prise sur le PN (tableau II, b), la fonction de von Bertalanffy a permis d'estimer les performances à âge type avec une perte de précision de 2 à 3 p. 100 pour P30, de 1 à 2,5 p. 100 pour $P 70$, de 4 à 11 p. 100 pour la valeur laitière (GMQ00/30 et GMQ10/30), et de 6 à 7 p. 100 pour le GMQ30/70. Les coefficients de régression des performances relatives aux protocoles simplifiés sur celles prises comme référence ont été peu différents de l'unité pour P30 ou P70, mais légèrement différents pour GMQ10/30, GMQ00/30 et GMQ30/70. La perte de précision associée à P10 a été de l'ordre de 40 p. 100 alors que le coefficient de régression a été largement différent de l'unité. Par rapport aux hypothèses concernant la prise en compte du poids à la naissance, les plus faibles pertes de précision ont été obtenues avec le PNR, les plus élevées avec un PNF et l'utilisation d'un PNE a donné des résultats intermédiaires. Il en a été de même pour le poids à la naissance. En effet, le tableau II, b montre les pertes de précision associées à ce caractère.

Dans le cas des intervalles naissance - premier contrôle inférieurs à 22 jours (tableau II, a), les pertes de précision sur l'estimation de l'ensemble des performances à âge type ont été élevées ; la valeur la plus faible a été associée à P30 et a été au mieux égale à 23 p. 100.

Quant aux intervalles supérieurs à 42 jours (tableau II, c), les pertes de précision sur l'ensemble des performances ont été plus faibles que celles du scénario précédent ; celles associées à P30 ont varié entre 17 et 19 p. 100, alors que la valeur la plus faible a été associée à P70 et de l'ordre de 4 p. 100. Toutefois, ces deux situations (tableau II, a et c) n'ont pas beaucoup d'intérêt globalement ; c'est au niveau du détail (perte de précision par jour d'intervalle naissance - premier contrôle) que pourraient être précisées les limites acceptables d'extension avant et après l'intervalle 22-42 jours.

A cet égard, dans l'intervalle naissance - premier contrôle allant de 6 à 22 jours, la perte de précision sur l'estimation de la valeur

\section{Tableau II}

Perte de précision associée à l'estimation des performances à âge type des jeunes agneaux de race Barbarine par l'ajustement de la fonction de von Bertalanffy à différents protocoles de contrôle de croissance simplifiés à deux pesées et au poids à la naissance réel, fixé ou estimé

\begin{tabular}{|c|c|c|c|c|c|c|}
\hline & \multicolumn{2}{|c|}{ PN réel } & \multicolumn{2}{|c|}{ PN fixé } & \multicolumn{2}{|c|}{ PN estimé } \\
\hline & $1-\mathbf{R}^{2}$ & $\mathbf{b}$ & $1-\mathbf{R}^{2}$ & b & $1-R^{2}$ & b \\
\hline \multicolumn{7}{|c|}{ a) Intervalle naissance - $1^{\text {er }}$ contrôle à $1-21$ jours } \\
\hline $\mathrm{PN}$ & 0,547 & 0,464 & 0,986 & 0,135 & 0,762 & 0,410 \\
\hline P10 & 0,290 & 0,873 & 0,325 & 0,792 & 0,365 & 0,825 \\
\hline P30 & 0,238 & 0,759 & 0,235 & 0,715 & 0,310 & 0,678 \\
\hline P70 & 0,294 & 0,711 & 0,324 & 0,676 & 0,390 & 0,586 \\
\hline GMQ10/30 & 0,270 & 0,962 & 0,373 & 1,070 & 0,651 & 0,949 \\
\hline GMQ0030 & 0,348 & 0,666 & 0,354 & 0,595 & 0,400 & 0,611 \\
\hline GMQ3070 & 0,649 & 0,458 & 0,691 & 0,425 & 0,652 & 0,431 \\
\hline \multicolumn{7}{|c|}{ b) Intervalle naissance - $1^{\mathrm{er}}$ contrôle à $22-42$ jours } \\
\hline $\mathrm{PN}$ & 0,121 & 0,923 & 0,996 & $-0,167$ & 0,480 & 1,271 \\
\hline P10 & 0,392 & 0,661 & 0,462 & 0,442 & 0,390 & 0,546 \\
\hline P30 & 0,026 & 0,991 & 0,031 & 1,012 & 0,022 & 1,036 \\
\hline P70 & 0,024 & 0,981 & 0,013 & 0,980 & 0,012 & 0,978 \\
\hline GMQ10/30 & 0,041 & 1,015 & 0,103 & 1,120 & 0,081 & 1,170 \\
\hline GMQ00/30 & 0,054 & 0,940 & 0,116 & 0,806 & 0,097 & 0,913 \\
\hline GMQ30/70 & 0,067 & 0,924 & 0,073 & 0,911 & 0,063 & 0,918 \\
\hline \multicolumn{7}{|c|}{ c) Intervalle naissance - $1^{\text {er }}$ contrôle à $43-60$ jours } \\
\hline $\mathrm{PN}$ & 0,207 & 0,846 & 0,998 & $-0,096$ & 0,525 & 1,167 \\
\hline P10 & 0,616 & 0,609 & 0,783 & 0,351 & 0,664 & 0,477 \\
\hline P30 & 0,173 & 0,920 & 0,191 & 0,976 & 0,172 & 0,973 \\
\hline P70 & 0,078 & 0,979 & 0,038 & 0,976 & 0,039 & 0,984 \\
\hline GMQ10/30 & 0,193 & 0,913 & 0,213 & 0,990 & 0,216 & 1,022 \\
\hline GMQ00/30 & 0,216 & 0,880 & 0,223 & 0,799 & 0,208 & 0,874 \\
\hline GMQ30/70 & 0,277 & 0,806 & 0,275 & 0,771 & 0,268 & 0,807 \\
\hline
\end{tabular}

PN : poids à la naissance ; P10 : poids à 10 jours ; GMQ10/30 : gains moyens quotidiens entre 10 et 30 jours 
laitière, exprimée en GMQ10/30 (figure 2), a été comparable au témoin (perte de précision associée au GMQ10/30 estimé par le protocole conventionnel avec PN contrôlé). Pour un intervalle inférieur à six jours, la perte de précision a été supérieure à 20 p. 100 et a dépassé le témoin. Pour un intervalle supérieur à 42 jours, les résultats des différentes simulations ont été comparables entre eux et avec le témoin jusqu'à 50 jours. Au-delà, le protocole simplifié a permis l'estimation de la valeur laitière avec une perte de précision comparable à celle associée au protocole conventionnel sans contrôle du poids à la naissance, et supérieure à la perte de précision relative au protocole conventionnel avec un poids à la naissance contrôlé.

Concernant l'estimation du GMQ30/70 (figure 3), la perte de précision a été au maximum égale à 10 p. 100 dans l'intervalle 22-42 jours et comparable entre les trois scénarios sur le poids à la naissance. En dehors de cet intervalle, elle a atteint 40 à 48 p. 100 quand l'intervalle est descendu à 15 jours et jusqu'à 22 p. 100 quand l'intervalle s'est élevé à 46 jours.

La perte de précision associée au calcul du P30, dans l'intervalle 22-42 jours (figure 4$)$ a été minimale $(0,1$ à 0,4 p. 100) vers 30 jours et a augmenté vers les deux extrémités pour atteindre 3 et 5 p. 100 respectivement à 22 et 42 jours d'intervalle. La différence pour le PN entre les trois scénarios a été encore une fois négligeable. En dehors de cet intervalle, la perte de précision a augmenté jusqu'à 7 p. 100 à 15 jours et 9 p. 100 à 45 jours d'intervalle.

Quelle qu'ait été l'hypothèse pour le PN, la perte de précision pour P70 (figure 5) est descendue sous les seuils de 10 p. 100 à 15 jours d'intervalle et de 3 p. 100 à 22 jours d'intervalle. Elle n'a pas été dépassée avant 44 jours, a augmenté légèrement pour atteindre un maximum de 5.5 p. 100 à 48 jours et est redescendue de nouveau.

\section{DISCUSSION}

L'ajustement d'une courbe de croissance à deux pesées à 42 jours d'intervalle et dont la première a été réalisée entre 22 et 42 jours après la naissance a permis de déduire les performances à âge type 10, 30 et 70 jours, et les gains (GMQ10/30 et GMQ30/70) sans grande perte de précision. La perte de précision associée au GMQ10/30 a été moindre, ne dépassant guère 12 p. 100, par rapport à celle obtenue avec le protocole conventionnel, qui a atteint 20 p. 100 à 30 jours d'intervalle naissance - premier contrôle. Les pertes de précision les plus élevées, associées à la simplification du contrôle laitier ovin (8), ont été de 10 à 15 p. 100 pour le calcul des poids à 30 et 70 jours. Concernant le GMQ30/70, déduit de ces derniers, elles ont été acceptables, inférieures à 20 p. 100 . Associées aux différentes performances, elles ont été légèrement inférieures à celles obtenues par l'ajustement d'une courbe de

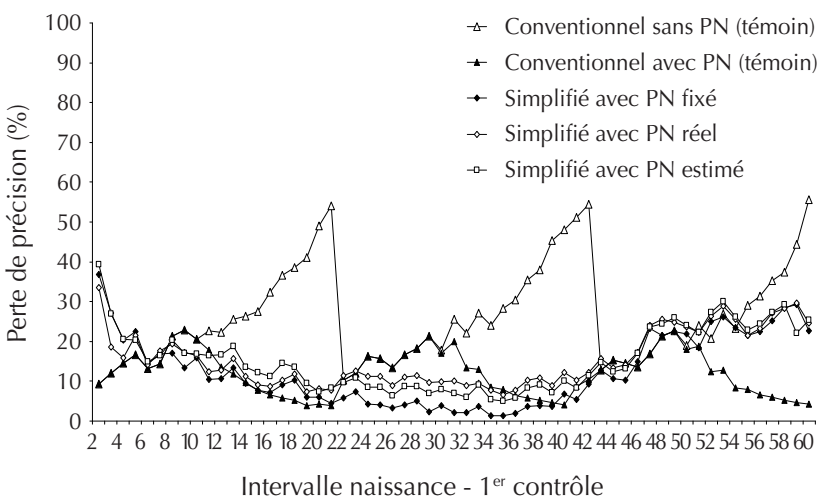

Figure 2 : perte de précision associée à l'estimation du GMQ10/30 selon un protocole simplifié à deux pesées et trois scénarios sur le poids à la naissance, réel, fixé et estimé (PN : poids à la naissance ; GMQ10/30 : gains moyens quotidiens entre 10 et 30 jours).

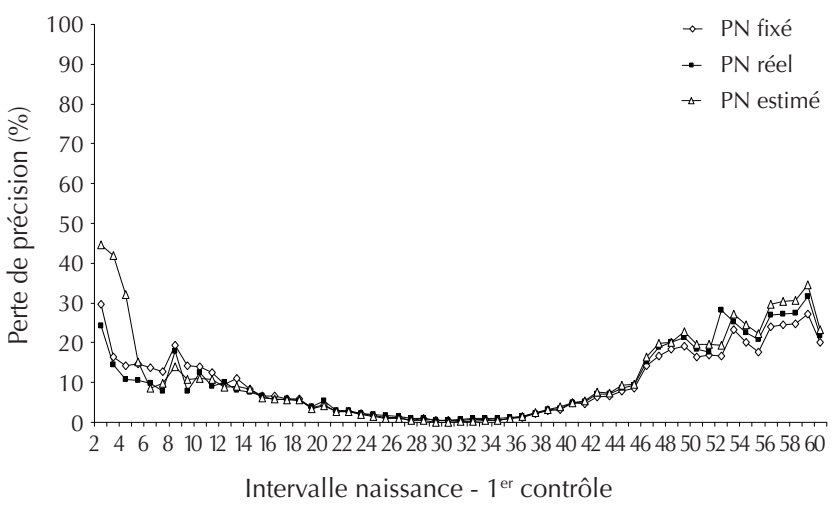

Figure 4 : perte de précision associée à l'estimation du poids à 30 jours selon un protocole simplifié à deux pesées et trois scénarios sur le poids à la naissance, réel, fixé et estimé.

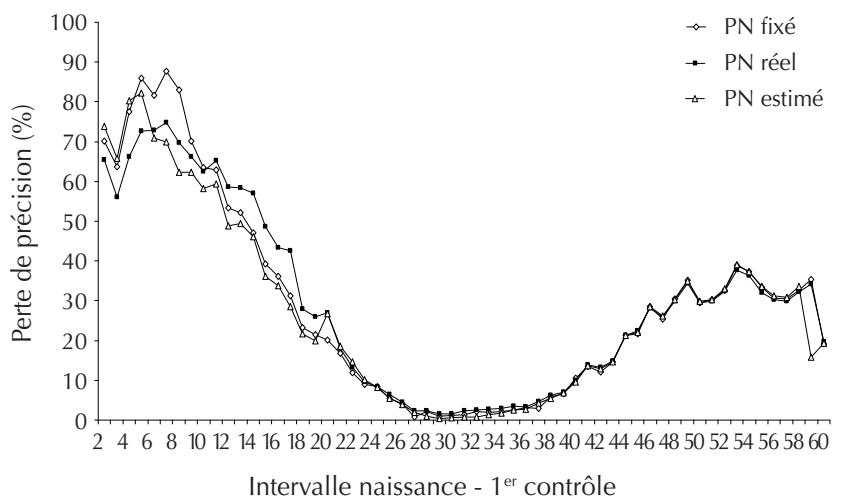

Figure 3 : perte de précision associée à l'estimation du GMQ30/70 selon un protocole simplifié à deux pesées et trois scénarios sur le poids à la naissance, réel, fixé et estimé (PN : poids à la naissance; GMQ10/30 : gains moyens quotidiens entre 30 et 70 jours).



Figure 5 : perte de précision associée à l'estimation du poids à 70 jours selon un protocole simplifié à deux pesées et trois scénarios sur le poids à la naissance, réel, fixé et estimé. 
croissance à trois contrôles et un poids à la naissance (3), et largement inférieures à celles relatives à l'ajustement d'une courbe de croissance à six contrôles et un poids à la naissance $(3,4)$. De fait, l'ajustement d'une courbe de croissance globale masque les variations à court terme (10). Ce phénomène a semblé, à travers les présents résultats, d'autant plus prononcé que le nombre de contrôles servant à l'ajustement de la courbe a été élevé.

Etant donné les résultats relatifs à la valeur laitière, les conclusions se rapportant à l'intervalle naissance - premier contrôle entre 22 et 42 jours sont restées valables dans l'intervalle 6-46 jours. En effet, le long de cet intervalle, la perte de précision associée à l'estimation de la valeur laitière a été équivalente, voire inférieure à celle déjà admise par le protocole conventionnel. La précision de l'estimation de la valeur laitière s'est donc améliorée malgré la simplification. La précision du calcul du GMQ30/70, en revanche, a semblé être affectée par la simplification ; elle est passée de 20 à 40 p. 100 quand l'intervalle naissance - premier contrôle est passé de 21 à 15 jours. Celle du poids à 70 jours l'a été moins ; elle est passée de 5 à 10 p. 100 pour la même variation de l'intervalle. Il faut préciser, cependant, que les intervalles naissance - premier contrôle de 21 et 15 jours correspondaient à une deuxième pesée, respectivement à 63 et 56 jours (intervalle P1-P2 de 42 jours), ce qui conduit à un calcul du poids à 70 jours par extrapolation dans la limite respectivement de 7 et 14 jours.

L'élaboration de propositions de contrôle de croissance alternatives au protocole officiel, basées sur l'ajustement d'une fonction non linéaire à un nombre réduit de contrôles et un poids à la naissance, doit découler des résultats du présent travail. Toutefois, le taux de perte de précision toléré sur le calcul des performances peut être lié à l'importance de chacune d'elles dans l'objectif de sélection ; ainsi :

- une première pesée, au plus tard 46 jours après la première naissance dans le troupeau, devrait constituer la base de toute proposition alternative ; ceci permettrait le calcul du GMQ10/30 ou du GMQ00/30 des agneaux nés au plus tard 15 jours avant la date du contrôle, avec une perte de précision inférieure à 15 p. 100, ou au plus tard six jours avant, avec une perte de précision inférieure à 20 p. 100. Il est important de rappeler que cette perte de précision est dans les deux cas équivalente, voire inférieure à celle admise par le protocole actuel F2. Ben Hamouda (3), et Tiphine et coll. (13) ont envisagé la suppression de la première visite et de la reporter à 42 jours après la première naissance dans le troupeau, alors que Ben Gara et coll. (2) ont déjà proposé depuis 1997 la possibilité de la retarder ;

- l'intervalle entre la première et la deuxième pesée peut être défini en fonction de la perte de précision à tolérer sur le calcul du caractère à associer à la valeur laitière, le P70 ou le GMQ30/70, dans l'objectif de sélection. A cet égard, la perte de précision, plus faible, associée à l'estimation du P70 plaide en faveur de son maintien comme critère de sélection, d'autant que son poids économique devrait être, pour une race allaitante, inférieur à celui de la valeur laitière. Or, le taux de perte de précision sur le calcul du P70 est lié, en particulier, à la marge d'extrapolation à admettre pour les agneaux n'ayant pas atteint l'âge de 70 jours lors de leur second contrôle. Pour rappel, aux marges d'extrapolation de 7 et de 14 jours ont été associés respectivement 5 et 10 p. 100 de perte de précision.

Les bases ainsi mises en place, il s'agit de revenir à l'objectif initial d'alléger le coût du contrôle de croissance des agneaux en conditions difficiles ; les scénarios « contrôle allégé » et « contrôle simplifié » ont été proposés.

\section{Contrôle allégé}

L'objectif recherché par ce protocole a été de réduire le coût sans altérer la précision du calcul des performances à âge type. Une première pesée à 46 jours du premier agnelage dans le troupeau a permis de calculer le GMQ10/30 ou GMQ00/30 des agneaux nés le premier mois, avec une perte de précision inférieure à 15 p. 100 . Soumettre les naissances du deuxième mois aux mêmes conditions implique une seconde visite à un mois de la première. A cette date, les agneaux nés le premier mois atteignent un âge compris entre 46 et 76 jours (tableau III) ; un troisième contrôle est donc nécessaire à la précision du calcul du P70 d'un grand nombre d'entre eux. Ce scénario se base sur trois pesées par agneau et améliore de manière significative, par rapport au protocole actuel, la précision de l'estimation de la valeur laitière alors que le calcul de P70 et du GMQ30/70 ne devrait pas être affecté.

\section{Contrôle simplifié}

Tolérer une perte de précision inférieure à 20 p. 100 sur l'estimation de la valeur laitière implique qu'une première pesée à

\section{Tableau III}

Principales règles et paramètres de deux protocoles allégés et simplifiés alternatifs au protocole conventionnel, pour le contrôle de croissance des agneaux en conditions difficiles

\begin{tabular}{|c|c|c|c|c|c|c|c|c|c|}
\hline Protocole & $\begin{array}{c}1^{\text {re }} \text { naissance } \\
-P_{1}\end{array}$ & $\begin{array}{l}\text { Rythme } \\
\text { de pesée }\end{array}$ & Performance & $\begin{array}{c}\text { Période } \\
\text { d'agnelage }\end{array}$ & $\begin{array}{l}\text { Int. nais. - } \\
\qquad \mathbf{P}_{1}^{* *}\end{array}$ & $\begin{array}{l}\text { Int. nais. - } \\
\qquad \mathbf{P}_{2}^{* *}\end{array}$ & $\begin{array}{l}\text { Int. nais. - } \\
\qquad \mathbf{P}_{3}^{* *}\end{array}$ & $\begin{array}{l}\text { Int. nais. - } \\
\qquad \mathbf{P}_{4}^{* *}\end{array}$ & $\begin{array}{cc}\text { Marge } & \text { Perte de } \\
\text { extrapolation } & * \\
\text { précision }(\%)\end{array}$ \\
\hline \multirow[t]{4}{*}{ Allégé } & 46 jours & 30 jours & GMQ00/30 & $01-30$ & $46-16$ & & & & $\leq 15$ \\
\hline & & & & $31-60$ & & $46-16$ & & & $\leq 15$ \\
\hline & & & P70 & $01-30$ & $46-16$ & $76-46$ & $106-76$ & & $<05$ \\
\hline & & & & $31-60$ & - & $46-16$ & $76-46$ & $106-76$ & $<05$ \\
\hline \multirow[t]{4}{*}{ Simplifié } & 46 jours & 40 jours & GMQ00/30 & $01-40$ & $46-06$ & & & & $\leq 25$ \\
\hline & & & & $41-70$ & - & $46-06$ & & & $\leq 25$ \\
\hline & & & P70 & $01-30$ & $46-16$ & $86-56$ & $126-96$ & & $<05$ \\
\hline & & & & $31-70$ & & $46-16$ & $86-56$ & & 14 jours \\
\hline
\end{tabular}

$P_{1}: 1^{\text {re }}$ pesée ; Int. nais. : intervalle naissance ; GMQ10/30 : gains moyens quotidiens entre 10 et 30 jours

* Extrapolation de P70 ; ** les limites de l'intervalle exprimées par rapport à la naissance du premier et du dernier agneau concerné par chaque contrôle 
46 jours servira à l'estimation du GMQ10/30 ou du GMQ00/30 des agneaux nés les premiers 40 jours de la saison d'agnelage. En outre, ceci permet de retarder le second contrôle de 10 jours et de passer à un intervalle de 40 jours entre les visites. A la date de la seconde visite, les agneaux nés durant les 30 premiers jours auront un âge compris entre 56 et 86 jours (tableau III) et l'extrapolation du P70 des agneaux les plus jeunes sera accompagnée d'une perte de précision de 10 p. 100. A la date d'une troisième visite, les agneaux nés le deuxième mois de la saison d'agnelage seront âgés de 66 à 86 jours ; leurs poids à 70 jours seront donc calculés avec une perte de précision ne dépassant pas les 5 p. 100. C'est un protocole qui nécessite deux pesées par agneau, donc allégé par rapport au précédent ; il peut être qualifié de simplifié. Il améliore l'estimation de la valeur laitière mais s'accompagne d'une perte de précision légère pour P70 et assez significative pour GMQ30/70. Par ailleurs et sans recourir à d'autres visites, le présent scénario peut tolérer une extension de la saison d'agnelage de 10 jours, soit une saison de 70 jours au lieu 60 jours dans le cas du contrôle allégé.

\section{CONCLUSION}

L'ajustement d'une fonction non linéaire à la croissance des agneaux durant les trois premiers mois de vie peut être réalisé à partir d'un poids à la naissance et de trois, voire deux pesées séquentielles. Cependant et à défaut d'un contrôle à la naissance, opération lourde et coûteuse, un poids à la naissance fixé, conforme au standard de la race ou estimé en fonction de la première pesée et l'intervalle qui la sépare de la naissance remplit la condition. Sur une telle courbe de croissance, la valeur laitière (GMQ10/30 ou GMQ00/30) est estimée avec une précision meilleure que celle obtenue avec la méthode conventionnelle ; l'estimation du P70 est accompagnée d'une perte de précision inférieure à celle associée au GMQ30/70. La première visite de contrôle de croissance peut avoir lieu 46 jours après la première naissance dans le troupeau au lieu de 21 jours actuellement. Le niveau de précision recherché sur le calcul des performances à âge type est déterminant dans la précision du nombre de pesées par agneau, deux ou trois, et le rythme des visites de contrôle. Une perte de précision maximale de 15 p. 100 sur l'estimation de la valeur laitière (GMQ10/30 ou GMQ00/30) et de 5 p. 100 pour P70 est obtenue par une première pesée à 46 jours du début des agnelages et deux autres à un mois d'intervalle. Tolérer 25 et 10 p. 100 de perte maximale sur les mêmes performances ramènerait le nombre de pesées à deux par agneau, une première à 46 jours du début des agnelages et une seconde à 40 jours de la première. Une analyse génétique apporterait davantage de précisions aux présentes conclusions.

\section{BIBLIOGRAPHIE}

1. BASDAGIANNI Z., BANOS G., ABS Z., ARZENOS G., VALERGAKIS G.E., ZYGOIANNIS D., 2005. Estimation of daily and total lactation milk yield of Chios ewes from single morning or evening records. Small Rum. Res., 92: 59-68.

2. BEN GARA A., ROUISSI H., JURADO J.J., BODIN L., GABINA D., BOUJENANE I., MAVROGENIS A.P., DJEMALI M., SERRADILLA J.M., 1997. Etude de la simplification du protocole de pesées chez les ovins à viande. Cah. Options méditerr. Sér. A, $33: 11-34$.

3. BEN HAMOUDA M., 2005. Minimum sequential records to adequatly fit growth curve in fat-tailed Barbarine lambs and implications on genetic evaluation. In: Proc. 34th Biannual Session of ICAR, Performance recording of animals, state of the art, Sousse, Tunisia, 28 May - 3 June. EAAP Publ., 113: 91-96.

4. BEN HAMOUDA M., 2012. Contrôle de croissance des ovins allaitants en Tunisie. I. Ajustement d'une courbe globale et analyse critique du protocole officiel. Rev. Elev. Méd. vét. Pays trop., 65 : 31-39.

5. BOUJENANE I., AIT BIHI N., BOUKALLOUCHE A., 1995. Amélioration génétique des ovins au Maroc. Cah. Options méditerr., 11 : 109-119.

6. BOULOC N., BARILLET F., BOICHARD D., SIGWALD J.P., BRIDOUX G., 1991. Etude des possibilités d'allégement du contrôle laitier officiel chez les caprins. Ann. Zootech., 40 : 125-139.

7. DE RANCOURT M., FOIS N., LAVIN M.P., TCHAKERIAN E., VALLERAND F., 2006. Mediterranean sheep and goat production: An uncertain future. Small Rum. Res., 62: 167-179.

8. GONZALO C., OTHMANE M.H., FUERTES I.A., DE LA FUENTE L.F., SAN PRIMITIVO F., 2003. Losses of precision associated with simplified designs of milk recording for dairy ewes. J. Dairy Res., 70: 441-444.

9. HOLST P.J., 1999. Recording and on-farm evaluations and monitoring: breeding and selection. Small Rum. Res., 34: 197-202.

10. LINDEN VAN DER F.P.G., HIRSCFIELD W.J., MILLER R.L., 1970. On the analysis and presentation of longitudinally collected data. Growth, 34: 385-400.

11. OTHMANE M.H., FUERTIS J.A., GONZALO C., DE LA FUENTI L.F., 2006. Accuracy of simplified sampling procedures for estimating milk composition in dairy ewes. J. Dairy Res., 73: 109-114.

12. SAS/STAT, 1996. Users' Guide, vers. 6.1. Cary, NC, USA, SAS Institute.

13. TIPHINE L., BOUIX J., POIVEY J.P., 2005. Proposition d'allègement du contrôle de performances en ovins allaitants. In : Actes $12^{\text {es }}$ Rencontres Recherches Ruminants, Paris, France, 7-8 déc. 2005, 349 p.

Accepté le 10.07.2012 


\section{Summary}

Ben Hamouda M., Rekik M. Growth monitoring of suckling lambs in Tunisia. II. Loss of precision in calculating performances at typical ages according to a simplified protocol as alternative to the conventional protocol

The performances at typical ages P10 (weight at 10 days), P30, P70, DWG10/30 (average daily weight gain between 10 and 30 days) and DWG30/70 of 9642 Barbarine lambs were calculated according to $\mathrm{F} 2$, the conventional protocol, or a simplified protocol based on fitting von Bertalanffy's function to two measurements at 42-day intervals, the first taking place between 2 and 60 days after birth of the lamb, and the actual birth weight (ABW: controlled at birth), a fixed BW $(\mathrm{FBW})$, i.e. the same for all lambs $(\mathrm{FBW}=3.4 \mathrm{~kg})$, or an estimated BW (EBW) based on the first measurement $(\mathrm{W})$ and the interval between birth and the first measurement (D) [(EBW = $2.71009+0.208313 W-0.00578 W(60-D)+0.00016 W$ $\left.(60-\mathrm{D})^{2}\right]$. The average loss of precision associated with the calculation of weights at typical ages was high for P10 (40$45 \%)$, moderate for P30 (6-7\%) and low for P70 (2-3\%). The loss of precision associated with DWG10/30 was lower than that of the conventional protocol for an interval between birth and the first control between 15 and 45 days ( $15 \%$ maximum), and similar between 7 and 15 days' intervals (20\% maximum). These results suggest simplifying the F2 protocol to two or three measurements and a birth weight. The first measurement should be postponed to 46 days after birth, whereas the interval between successive controls may vary between 30 and 40 days, depending on an acceptable loss of precision on the estimation of performances at typical ages.

Keywords: Sheep - Birth weight - Growth control - Alternative method - Tunisia.

\section{Resumen}

Ben Hamouda M., Rekik M. Control del crecimiento de ovejas lactantes, en Túnez. II. Pérdida de precisión en el cálculo del rendimiento por edad estándar según un protocolo simplificado alternativo al protocolo oficial

El rendimiento en la edad estándar de P10 (10 días), P30, P70, GMD10/30 (ganancia media diaria entre 10 y 30 días) y GMD30/70 de 9.642 corderos de raza Barbarine se calculó según el protocolo convencional «F2» o un protocolo simplificado basado en el ajuste de la función von Bertalanffy en dos pesajes con 42 días de intervalo, el primero tiene lugar entre 2 y 60 días después del nacimiento del cordero, y un peso al nacimiento real (PNR: controlado al nacimiento), fijado, el mismo para todos los corderos ( $P N F=3,4 \mathrm{~kg}$ ), o estimado en función del primer pesaje $(\mathrm{P})$ y el intervalo entre el nacimiento y el primer pesaje (D) $[\mathrm{PNE}=2,71009+0,208313$ $\left.\mathrm{P}-0,00578 \mathrm{P}(60-\mathrm{D})+0,00016 \mathrm{P}(60-\mathrm{D})^{2}\right]$. El promedio de pérdida de precisión asociada al cálculo de los pesos a la edad estándar fue elevado para el P10 (40 a 45\%), moderado para el P30 (6 a 7\%) y bajo para el P70 (2 a 3\%). La pérdida de precisión asociada a la GMD10/30 fue inferior a aquella previamente admitida por el protocolo convencional para un intervalo entre el nacimiento y el primer control de 15 a 45 días (máximo 15\%), y semejante entre 7 y 15 días de intervalo (máximo 20\%). Estos resultados permitieron proponer la simplificación del protocolo F2 en dos o tres pesajes y un peso al nacimiento. El primer pesaje debería retrasarse hasta 46 días después del nacimiento cuando el intervalo entre controles sucesivos puede variar entre 30 y 40 días, dependiendo de la pérdida de precisión que puede ser tolerada en la estimación del rendimiento por edad estándar.

Palabras clave: Ovino - Peso al nacimiento - Control del crecimiento - Método alternativo - Túnez. 
